\title{
The Belgian trial with azithromycin for acute COPD exacerbations requiring hospitalization: an investigator-initiated study protocol for a multicenter, randomized, double-blind, placebo- controlled trial
}

This article was published in the following Dove Press journal:

International Journal of COPD

31 March 2016

Number of times this article has been viewed

Kristina Vermeersch,' Maria Gabrovska, ${ }^{2}$ Griet Deslypere, ${ }^{3}$ Ingel K Demedts, ${ }^{4}$ Hans Slabbynck, ${ }^{5}$ Joseph Aumann, ${ }^{3}$ Vincent Ninane, ${ }^{2}$ Geert M Verleden,' Thierry Troosters, ${ }^{1,6}$ Kris Bogaerts, ${ }^{7,8}$ Guy G Brusselle, ${ }^{9}$ Wim Janssens'

On behalf of the BACE Trial Investigators

'KU Leuven, Laboratory of Respiratory Diseases, Department of Clinical and Experimental Medicine, Faculty of Medicine, Leuven, Belgium; ' ${ }^{2}$ epartment of Pneumology, Centre Hospitalier Universitaire Saint-Pierre, Brussels, Belgium; ${ }^{3}$ Department of Pneumology, Jessa Ziekenhuis, Hasselt, Belgium; ${ }^{4}$ Department of Respiratory Medicine, AZ Delta Roeselare-Menen, Roeselare, Belgium; ${ }^{5}$ Department of Respiratory Medicine, ZNA Middelheim, Antwerpen, Belgium; ${ }^{6} \mathrm{KU}$ Leuven, Department of Rehabilitation Sciences, Faculty of Kinesiology and Rehabilitation Sciences, Leuven, Belgium; ${ }^{7} \mathrm{KU}$ Leuven, Department of Public Health and Primary Care, I-BioStat, Leuven, Belgium; ${ }^{8} \mathrm{Hasselt}$ University, Hasselt, Belgium; ${ }^{9}$ Department of Respiratory Medicine, Ghent University Hospital, Ghent, Belgium

Correspondence: Wim Janssens KU Leuven, Laboratory of Respiratory Diseases, Department of Clinical and Experimental Medicine, Herestraat 49, O\&NI, Box 706, B-3000 Leuven, Belgium

$\mathrm{Tel}+3216346812$

$\mathrm{Fax}+3216346803$

Email wim.janssens@uzleuven.be
Background: Long-term use of macrolide antibiotics is effective to prevent exacerbations in chronic obstructive pulmonary disease (COPD). As risks and side effects of long-term intervention outweigh the benefits in the general COPD population, the optimal dose, duration of treatment, and target population are yet to be defined. Hospitalization for an acute exacerbation (AE) of COPD may offer a targeted risk group and an obvious risk period for studying macrolide interventions. Methods/design: Patients with COPD, hospitalized for an AE, who have a smoking history of $\geq 10$ pack-years and had $\geq 1$ exacerbation in the previous year will be enrolled in a multicenter, randomized, double-blind, placebo-controlled trial (NCT02135354). On top of a standardized treatment of systemic corticosteroids and antibiotics, subjects will be randomized to receive either azithromycin or placebo during 3 months, at an uploading dose of $500 \mathrm{mg}$ once a day for 3 days, followed by a maintenance dose of $250 \mathrm{mg}$ once every 2 days. The primary endpoint is the time-to-treatment failure during the treatment phase (ie, from the moment of randomization until the end of intervention). Treatment failure is a novel composite endpoint defined as either death, the admission to intensive care or the requirement of additional systemic steroids or new antibiotics for respiratory reasons, or the diagnosis of a new AE after discharge.

Discussion: We investigate whether azithromycin initiated at the onset of a severe exacerbation, with a limited duration and at a low dose, might be effective and safe in the highest risk period during and immediately after the acute event. If proven effective and safe, this targeted approach may improve the treatment of severe AEs and redirect the preventive use of azithromycin in COPD to a temporary intervention in the subgroup with the highest unmet needs.

Keywords: COPD, acute exacerbation, macrolide antibiotics, azithromycin, physical activity, RCT

\section{Introduction}

Chronic obstructive pulmonary disease (COPD) is one of the major health challenges of the next decades and is currently estimated by Global Burden of Disease studies as the third leading cause of death worldwide. ${ }^{1}$ It is a chronic disease characterized by airflow limitation which is progressive, not fully reversible and associated with an abnormal inflammatory response of the lung to noxious particles and gases. ${ }^{2-4}$ The high burden of COPD resulting from its respiratory symptoms is further enhanced by recurrent exacerbations, ${ }^{5,6}$ which are triggered mainly by the acquisition of new strains 
of respiratory pathogens or an increase in the concentration of bacteria colonizing the lower airways. ${ }^{7-9}$ Known to drive progressive lung function decline and physical inactivity, these acute events contribute significantly to morbidity and mortality, and the resulting hospitalizations impose a significant strain on health care resource utilization. ${ }^{10,11}$

Although the spectrum of available COPD treatments reduces the frequency of exacerbations by approximately $20 \%-30 \%,{ }^{12-15}$ they are insufficient as many patients still experience at least one exacerbation a year of which onequarter requires hospitalization. A recent large European audit on COPD exacerbations in hospitalized patients revealed that these events were associated with $12 \%$ mortality and $35 \%$ risk of readmission within 3 months after discharge. ${ }^{16}$ It is needless to argue that our current acute interventions are lacking effectiveness in a substantial proportion of the COPD admissions and that new interventions in this particular subset of patients are warranted. Bacterial infections are responsible for around half of the acute exacerbations (AEs) of COPD.${ }^{17}$ Current guidelines therefore recommend antibiotic therapy for patients with more severe symptoms, with treatment typically lasting 5-7 days. Although such intervention has shown to reduce the risk of subsequent exacerbations, relapse is common with some patients remaining at high risk for recurrent episodes for several weeks after the initial event. Failure may be related to inadequate antibiotic efficacy, but even with effective bacterial eradication, or in case of noninfectious events, increased airway inflammation may persist for a prolonged period after discharge and will likely promote recurrence. ${ }^{9}$ Epidemiological data confirm that the risk for subsequent events peaks drastically during 90 days after discharge and clearly increases with every following hospital admission. ${ }^{18}$ Disrupting the vicious cycle of one severe exacerbation introducing a subsequent one is therefore a promising interventional strategy.

Several randomized clinical trials have shown benefits of long-term macrolide treatment in a variety of chronic respiratory diseases, particularly in diffuse panbronchiolitis, cystic fibrosis, bronchiolitis obliterans syndrome post-lung transplantation, and bronchiectasis. ${ }^{19-23}$ The potential of long-term macrolide treatment for preventing COPD exacerbations has been recently confirmed in a large randomized controlled trial showing that chronic azithromycin therapy over 1 year, on top of standard inhalation therapy, reduced the risk of exacerbations by $\sim 30 \%{ }^{24}$ However, the long-term use of this class of antibiotics is not recommended in all patients with COPD as it is inevitably associated with bacterial resistance and carries the risk for side effects such as hearing loss and life-threatening arrhythmias by the prolongation of the
QT interval. ${ }^{25}$ Despite its potent effects and its proof of concept, uncertainty remains about the specific patient population that is most likely to benefit and also about the optimal dose and duration of the macrolide treatment. ${ }^{26}$ For these reasons, a recent update of the international treatment recommendations for COPD by the Global Initiative for Chronic Obstructive Lung Disease (GOLD) still did not incorporate azithromycin in its therapeutic arsenal. ${ }^{4}$ Reducing the dose and time course of the azithromycin intervention, as well as restricting the treatment to a subgroup of patients with COPD with the highest risk for future exacerbations, may overcome some of these concerns, and would therefore be a major step forward to a targeted clinical use. ${ }^{25,27}$ Additionally, short courses of macrolides were shown to facilitate the weaning process in ventilator-associated pneumonia and to shorten the time to resolution of pneumonia by approximately $40 \%{ }^{28}$ In AEs of asthma, 10 days of neomacrolides resulted in a significant symptom improvement compared to placebo. ${ }^{29}$ In line with these immediate therapeutic benefits for other acute respiratory diseases, azithromycin therapy, initiated at the onset of acute COPD exacerbations requiring hospital admission, may also improve short-term outcomes of hospitalization and prevent early relapse.

In this randomized, double-blind, placebo-controlled trial, the main aim is to assess whether a targeted course of low-dose azithromycin for 3 months, initiated at the onset of a severe AE requiring hospitalization, might be effective and safe in the highest risk period, that is, during and immediately after the acute event.

\section{Content}

This paper describes the BACE (the Belgian trial with azithromycin for acute COPD exacerbations requiring hospitalization; clinicaltrial.gov number: NCT02135354) study design and its novel contributions to the current clinical research in the management of AEs of COPD. These include a study protocol designed to be embedded in a real-life hospitalization setting following an AE, assessing the efficacy and safety of a dose- and time-limited treatment with azithromycin for prevention in the highest risk period, not only during but also immediately after the acute event. Furthermore, as a primary endpoint, we evaluate the time-to-treatment failure, a novel combined clinical endpoint defined as either death, the admission to intensive care or requirement of additional systemic steroids or new antibiotics for respiratory reasons, or the diagnosis of a new AE after discharge.

\section{Methodology}

Study design

The BACE trial is an investigator-initiated, multicenter, randomized, double-blind, placebo-controlled trial. Besides the 
lead center at the University Hospital in Leuven, patients will also be recruited from six academic and 13 nonacademic hospitals within Belgium. A maximum total of 500 patients will be enrolled by the established consortium within a 2-year inclusion period. The first patient was included in August 2014. The end of the trial is defined by the last visit of all included patients.

\section{Study population}

All patients with COPD, 18 years or older, hospitalized for an $\mathrm{AE}$ and treated with standard therapy, will be asked to participate in the BACE trial.

In order to observe changes in the treatment (or treatment failure) during and after hospitalization, the GOLDrecommended therapy during an $\mathrm{AE}$ requiring hospitalization (which includes systemic steroids, antibiotics, and shortacting bronchodilators) ${ }^{30}$ was fixed to a standard regimen (Table 1). This regimen is in line with recent literature on the dose of systemic steroids ${ }^{31-33}$ and the Infectious Diseases Advisory Board Belgian guidelines for antibiotic use in AEs of COPD requiring hospitalization.

\section{Inclusion and exclusion criteria}

All patients must have an established diagnosis of COPD made by a medical doctor (based on clinical history and a pulmonary function test), with a history of $\geq 1$ exacerbation in the preceding year for which a course of systemic steroids and/or

Table I Fixed regimen of standard therapy for an acute COPD exacerbation requiring hospitalization

\begin{tabular}{|c|c|}
\hline Therapy & Specifications \\
\hline \multirow[t]{2}{*}{ Systemic steroids } & Methylprednisolone 40 mg IV or 32 mg PO OD \\
\hline & for 5 days (switch IV to $\mathrm{PO}$ as soon as possible) \\
\hline \multicolumn{2}{|l|}{ Antibiotics } \\
\hline \multirow[t]{3}{*}{ First choice } & Amoxi-Clavulanate I g IV QID or 2 g PO BID \\
\hline & for 7 days (or alternative regimen of I g IV QID \\
\hline & or $875 / 125$ mg PO TID for 7 days) \\
\hline \multirow[t]{2}{*}{ Alternatives } & Moxifloxacin $400 \mathrm{mg}$ IV or $400 \mathrm{mg}$ PO OD for \\
\hline & 5 days \\
\hline \multirow[t]{4}{*}{ In case of: } & Intolerance or allergy to Amoxi-Clavulanate \\
\hline & Clinical failure on GP-initiated Amoxi-Clavulanate \\
\hline & treatment \\
\hline & Anti-pseudomonas antibiotics \\
\hline \multirow[t]{4}{*}{ In case of: } & Bronchiectasis \\
\hline & History of positive cultures for pseudomonas \\
\hline & High risk of pseudomonas \\
\hline & Clinical failure on GP-initiated treatment \\
\hline Short-acting & Via inhalation \\
\hline \multicolumn{2}{|l|}{ bronchodilators } \\
\hline \multirow[t]{3}{*}{ Respiratory support } & Oxygen \\
\hline & Noninvasive ventilation $^{\mathrm{a}}$ \\
\hline & Mechanical ventilation $^{a}$ \\
\hline
\end{tabular}

Note: ${ }^{a}$ Considered exclusion criteria if needed at the moment of randomization. Abbreviations: COPD, chronic obstructive pulmonary disease; IV, intravenous; PO, per os; OD, once a day; QID, four times a day; BID, two times a day; TID, three times a day; GP, general practitioner. antibiotics was started, must be current or past smokers with a smoking history of $\geq 10$ pack-years, and must have a normal QTc value confirmed by an electrocardiogram taken at admission. Automated QTc values, calculated according to Bazett's formula, and predefined cutoff criteria, that is, $\leq 450 \mathrm{~ms}$ for male and $\leq 470 \mathrm{~ms}$ for female, were used to determine the eligibility. The same formula was used throughout the patient's trial participation to evaluate QT prolongation.

The main exclusion criteria are contraindications to azithromycin, respiratory insufficiency at the moment of randomization, chronic systemic steroid use ( $>4 \mathrm{mg}$ methylprednisolone/day for $\geq 2$ months), and the active use of macrolide antibiotics during $\geq 2$ weeks preceding inclusion.

The full list of exclusion criteria is given in Table 2 .

\section{Intervention}

Eligible patients consenting to participation will be randomized (1:1) to receive either azithromycin (Azitromycine CF; Centrafarm Nederland B.V., Etten-Leur, the Netherlands) or placebo tablets of identical appearance (Apotheek Haagse Ziekenhuizen, Den Haag, the Netherlands) on top of maximal standardized therapy. Within 48 hours after hospital admission, a 3-month treatment phase with azithromycin or placebo is initiated at an uploading dose of $500 \mathrm{mg}$ once a day for 3 days and subsequently administered at a lower maintenance dose of $250 \mathrm{mg}$ every 2 days for the remainder of the 90-day

Table 2 Full list of exclusion criteria

\begin{tabular}{|c|c|}
\hline \multicolumn{2}{|c|}{ Exclusion criteria } \\
\hline 1 & $\begin{array}{l}\text { Mechanical or noninvasive ventilation at the moment } \\
\text { of randomization }\end{array}$ \\
\hline 2 & $\begin{array}{l}\text { Long QT interval on ECG: QTc }>450 \mathrm{~ms} \text { for male or }>470 \mathrm{~ms} \\
\text { for female }\end{array}$ \\
\hline 3 & History of life-threatening arrhythmias \\
\hline 4 & $\begin{array}{l}\text { Myocardial infarction (NSTEMI or STEMI) less than } 6 \text { weeks } \\
\text { before starting the study drug }\end{array}$ \\
\hline 5 & $\begin{array}{l}\text { Unstable angina pectoris or acute myocardial infarction } \\
\text { (NSTEMI or STEMI) at admission }\end{array}$ \\
\hline 6 & $\begin{array}{l}\text { Concomitant use of a drug with high risk for long QT interval and } \\
\text { torsade de pointes (amiodarone, flecainide, procainamide, sotalol, } \\
\text { droperidol, haldol, citalopram, other macrolides) }\end{array}$ \\
\hline 7 & $\begin{array}{l}\text { Documented uncorrected severe hypokalemia }\left(\mathrm{K}^{+}<3.0 \mathrm{mmol} / \mathrm{L}\right) \\
\text { or hypomagnesemia }\left(\mathrm{Mg}^{2+}<0.5 \mathrm{mmol} / \mathrm{L}\right)\end{array}$ \\
\hline 8 & $\begin{array}{l}\text { Chronic systemic steroids ( }>4 \text { mg methylprednisolone/day } \\
\text { for } \geq 2 \text { months) }\end{array}$ \\
\hline 9 & Actual use of macrolides for at least 2 weeks \\
\hline 10 & Allergy to macrolides \\
\hline II & Active cancer treatment \\
\hline 12 & Life expectancy $<3$ months \\
\hline 13 & $\begin{array}{l}\text { Pregnant or breast-feeding subjects. Woman of childbearing } \\
\text { potential must have a pregnancy test performed and a negative } \\
\text { result must be documented before starting the treatment }\end{array}$ \\
\hline
\end{tabular}

Abbreviations: ECG, electrocardiogram; NSTEMI, non-ST elevation myocardial infarction; STEMI, ST elevation myocardial infarction. 
treatment period. Patients will then be followed up for an additional 6 months to evaluate the recurrence rate after the withdrawal of study drug.

The BACE trial study design is presented in Figure 1.

\section{Rationale for duration and dose of azithromycin}

In 2011, Albert et al showed that azithromycin reduces the risk of future COPD exacerbations by $\sim 30 \%$ on top of standard inhalation therapy when given chronically for over a year at a dose of $250 \mathrm{mg}$ once daily. Careful observation of the data revealed that a major part of the reduction in exacerbations is already obtained within 90 days after treatment onset. Thereafter, the curves representing the proportion of patients free from AEs may still diverge, whereas after 6 months of treatment, a more steady and equal decline for both the placebo and intervention arm are reached. ${ }^{24}$ Remarkably, in the study by Wong et al on the reduction of exacerbations in bronchiectasis, in which azithromycin was stopped after 6 months of treatment, benefits persisted for another 6 months of follow-up. ${ }^{23}$ It indicates that the withdrawal of azithromycin after prolonged intake of 3-6 months is reasonable to consider and sufficient for interrupting the circle of inflammation, infection, and relapse.

In addition to the optimal duration for the azithromycin intervention, uncertainty remains about the ideal dose to target therapeutic serum and tissue levels. ${ }^{26}$ Earlier pharmacokinetic studies have found that a dose of $500 \mathrm{mg}$ corresponded to serum peak levels of $0.4 \mu \mathrm{g} / \mathrm{mL}$ with a half-life of 40-68 hours. With repeated administration, lung tissue levels increased 75-fold and persisted even after serum levels declined. ${ }^{34,35}$ Therefore, several experts believe that $250 \mathrm{mg}$ of azithromycin three times weekly is probably sufficient to obtain all therapeutic benefits while reducing potential side effects, although there are no specific data to support this in COPD. ${ }^{25,36}$

\section{Objectives and endpoints}

The primary objective is to prove the effectiveness of azithromycin on top of maximal standardized therapy in the acute treatment of COPD exacerbations that require hospitalization. A secondary objective is to prove the safety, without losing effectiveness, by reducing the dose and duration of a current and everlasting treatment.

The primary clinical efficacy endpoint is the time-totreatment failure and will be evaluated within the period from randomization (day 1, from 1 hour after the first intake of study drug) until the end of intervention (day 90, 24 hours after the last intake of study drug). By using this endpoint, the potential short-term benefits of acute macrolide treatment, as well as the long-term benefits of prolonged treatment, are utilized. The combined endpoint is defined as either death, the admission to intensive care

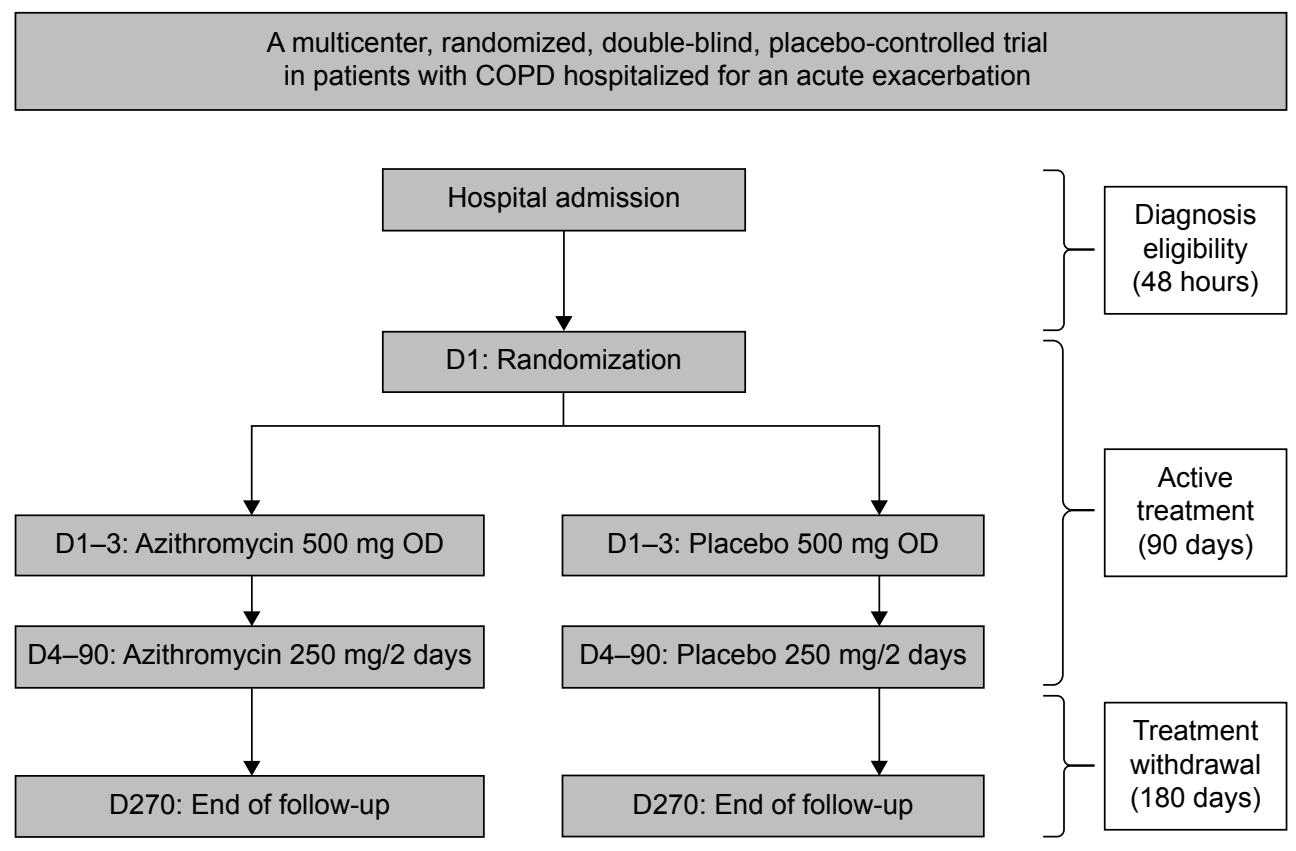

Figure I The BACE trial study design.

Abbreviations: BACE, the Belgian trial with azithromycin for acute COPD exacerbations requiring hospitalization; COPD, chronic obstructive pulmonary disease; DI, day I; DI-3, days I-3; D4-90, days 4-90; D270, day 270; OD, once a day. 
or the requirement of additional systemic steroids or new antibiotics for respiratory reasons, or the diagnosis of a new AE of COPD after discharge. Each of the components in the combined endpoint bears the same weight; once one is fulfilled, the primary endpoint is met. The use of this combined endpoint of treatment failure in the first 3 months is in line with earlier studies on the efficacy of systemic steroids during the $\mathrm{AE}^{37}$ and will avoid arbitrary clinical decisions on whether recurrent symptoms or therapy needed is related to an incomplete resolution or to a new acute event.

Key secondary endpoints are the number of clinical failures at day 90, symptom COPD Assessment Test (CAT) score at day 90, and total days of steroid use at day 90. Other main secondary endpoints are the key secondary endpoints at day 270 and endpoints assessed at the end of intervention (day 90) and the end of follow-up (day 270) including time to new exacerbation, number of new exacerbations, total days of hospitalization, total days of intensive care, quality-of-life (European Quality-of-Life - 5 Dimensions [EQ5D] questionnaire) and symptom assessments (CAT, modified Medical Research Council, and the Speech, Spatial, and Qualities of Hearing Scale - 5 Items questionnaires), forced expiratory volume in 1 second, total dose of systemic steroids, total days of antibiotic use, number of home physician contacts, and average costs of hospitalization.

\section{Randomization and masking}

Patients will be randomly assigned in a 1:1 ratio to receive either azithromycin or placebo, with a permuted block size of ten and sequential assignment, stratified by the center. Randomization and distribution of the study drug are performed by the hospital pharmacy of the University Hospital of Ghent based on an online generated randomization schedule (http://www.randomization.com). Unique randomization codes are locally obtained through a secured Web-based program. Participants, investigators, and research assistants are blinded to treatment allocation.

\section{Clinical procedures}

The study protocol is designed to be embedded in a real-life hospitalization setting following an AE. All screening tests to assess eligibility are part of the routine assessment in the emergency department. After initiating the intervention, study visits are scheduled to coincide with clinical routine practice in Belgium.

The study consists of the following visits that occur during hospitalization: randomization (day 1 is the first day of study drug administration), start of maintenance dose (day 4, +3-day window allowed for all actions with the exception of switching from the uploading to the maintenance dose), and day of discharge (day $X$, left at investigators discretion). After discharge, subjects will be followed at the outpatient department at 1 month after discharge (day $X+28,+14$-day window), the end of intervention (day 90, allowed from day 86 up to day 105), and the end of follow-up (day 270, +14-day window). Telephone calls are scheduled bimonthly (day 150 and day $210, \pm 7$-day window) between the end of intervention and the end of follow-up. Patients are instructed to complete a diary with biweekly questionnaires for symptom evaluation, medication changes and the detection of new exacerbations, general practitioner visits, or new hospitalizations. Adherence is assessed by study drug accountability and diary completion. Data collected at each visit are shown in Table 3. Performed in addition to clinical routine are the following tests: for the assessment of the impact of COPD - CAT questionnaire; level of dyspnea - modified Medical Research Council questionnaire; quality of life - EQ5D questionnaire; antibiotic resistance - bacterial and fungal culture on spontaneous sputum samples; cardiac toxicity - electrocardiogram; and the screening of hearing decrement - the Speech, Spatial and Qualities of hearing scale - 5-item questionnaire.

\section{Substudies PROactive substudy}

During and after AEs, patients with COPD are profoundly inactive and failure to increase physical activity (PA) is associated with relapse. Moreover, physical inactivity is known to be associated with cardiovascular and metabolic morbidity and is one of the strongest predictors of mortality in COPD. ${ }^{6}$ Recurrent AEs have a deleterious impact on PA and treatments implemented to reduce the burden of an $\mathrm{AE}$ may spin off in faster recovery of PA.

In a subgroup of the BACE trial, PA levels will be addressed with easy-to-wear portable devices $\left(\right.$ DynaPort $^{\mathbb{}}$; McRoberts B.V., the Hague, the Netherlands), recently thoroughly validated by the PROactive consortium for the use in COPD.$^{38}$ We hypothesize that with an effective medical intervention, differences in the recovery of PA will be appreciated when measuring 30 subjects in each treatment arm, offering considerable benefits in the long run.

Randomized patients willing to participate in the substudy will not only follow the standard protocol but also wear the activity monitor for 7 days after discharge (day $X$ ), at the end of intervention (day 90) and at the end of follow-up (day 270). This device is not only registering PA for 7 days but is also 


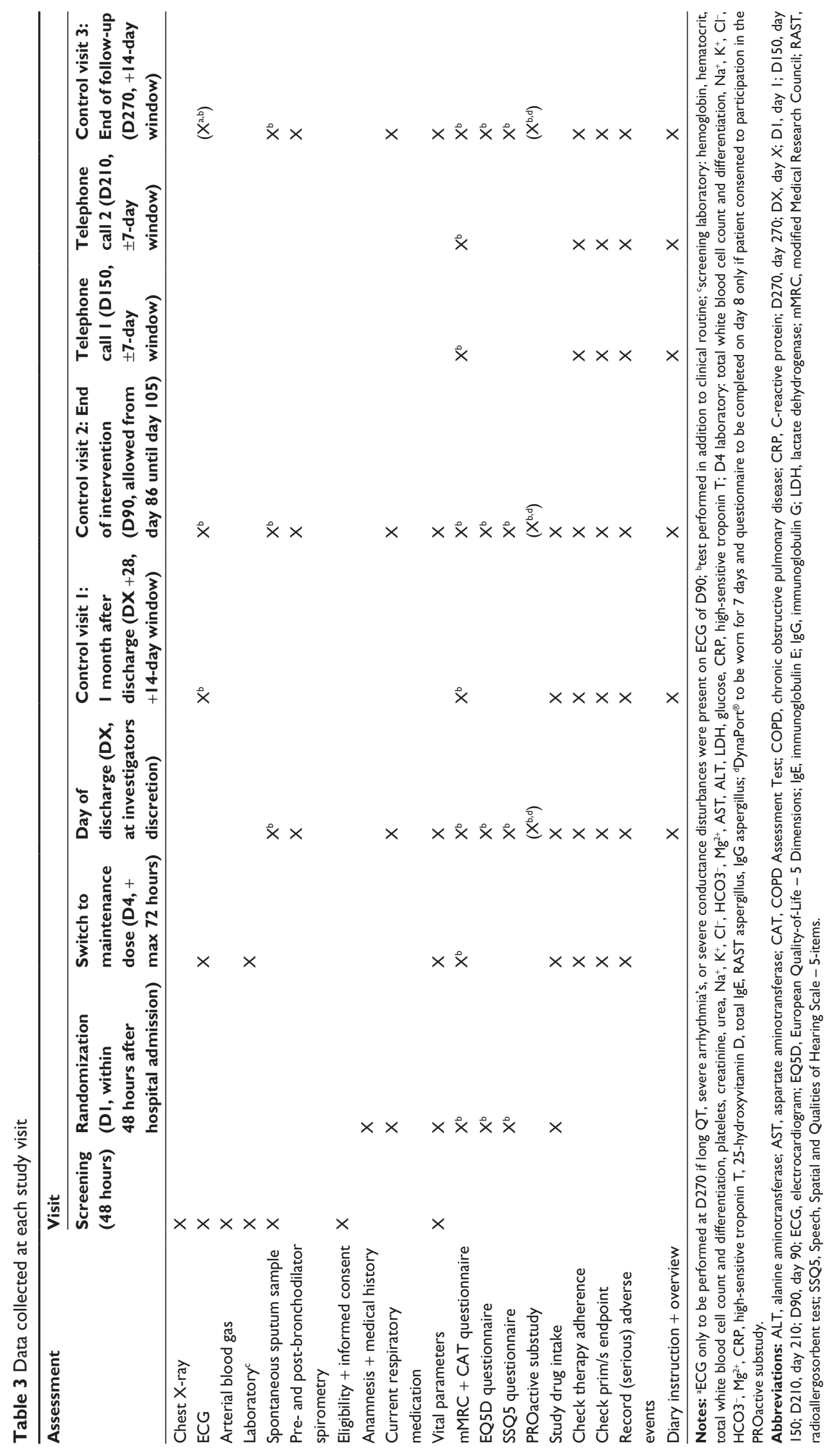


coupled to a standardized and patient-validated questionnaire on physical activities, which needs to be filled out at day 8 to cover a recall period of 7 (monitored) days.

\section{Pharmacoeconomic substudy}

A second subanalysis of the BACE trial will include a detailed cost-effectiveness study. This multicenter randomized trial executed in one country will provide an excellent tool for precise health economic assessments. In a first approach, rough estimates on savings of direct costs in the entire study cohort will be made by an evaluation of average costs in Flanders for a single hospitalization day at a respiratory ward, a day at intensive care, an emergency visit, a home physician contact, and for an antibiotic and/or steroid course. A more detailed cost-effectiveness and cost-utility analysis at 3-month and 9-month intervals will only be performed in case significant clinical benefits are found in favor of the active treatment.

For this purpose, medical resource use data will be collected not only retrospectively via hospital invoices (direct costs including medications, physician visits, laboratory tests, technical examinations, medical imaging, and hospital stay) but also prospectively via patient diaries, to cover direct and indirect costs (relating to time, convenience, and transportation) during the entire outpatient period and will be linked to the EQ5D scores.

\section{Ethical approval}

The BACE trial is being carried out according to the Good Clinical Practice Guidelines and the principles of the Declaration of Helsinki. Documented approval has been obtained from the Leading Ethics Committee (Commissie Medische Ethiek UZ KU Leuven, Leuven, Belgium - affiliated to UZ KU Leuven) and the competent authority (Federaal Agentschap voor Geneesmiddelen en Gezondheidsproducten [Federal Agency for Medicines and Health Products], Brussels, Belgium). All patients must provide a written informed consent according to the International Conference on Harmonization Good Clinical Practice Guidelines and local legal requirements. Randomized patients, willing to participate in the PROactive and/or pharmacoeconomic substudy, will have to give an informed consent for the additional measures related to either of the substudies. However, individual patients can choose to opt out for these measures and only to participate in the medical intervention study, the BACE trial.

\section{Statistics}

\section{Efficacy population}

The primary endpoint will be evaluated in the intentionto-treat population, that is, all randomized patients.
As a secondary analysis, the primary endpoint will also be evaluated in the per-protocol population (not including dropouts and including only patients with satisfactory adherence to the intake of the study drug).

\section{Sample size calculation}

Two hundred and fifty subjects per group, 500 subjects in total, will yield $80 \%$ power to show a significant difference in the primary endpoint at a two-sided significance level of 0.05. Sample size calculation is based on a survival analysis using a logrank test assuming proportional hazards, a clinical failure in at least $45 \%$ of the placebo arm within 3 months (based on 35\% readmissions in the Belgian COPD population in the European COPD audit, ${ }^{16}$ a proportion of $50 \%$ with a new exacerbation within 4 months after randomization in the randomized trial with vitamin D enrolling similar but stable patients, ${ }^{39}$ and a $20 \%$ treatment failure within 8 weeks in the MAESTRAL study looking at mild exacerbations $\mathrm{s}^{40}$ ), a $35 \%$ relative improvement with azithromycin intervention (hazard ratio $(\mathrm{HR})=0.65$; $\mathrm{HR}$ of 0.73 for time to first exacerbation, ${ }^{24} \mathrm{HR}$ of 0.5 for time to the resolution of ventilator-associated pneumonia ${ }^{28}$ ), and taking into account a maximal amount of $25 \%$ of dropouts. After the inclusion of 125 patients, recruitment was somewhat lower than anticipated and the observed dropout was also lower (16/125 [12.8\%]). Therefore, it was opted to foresee an interim analysis for efficacy and futility when 300 patients reach their 90-day follow-up. Assuming a lower dropout rate of $17 \%$ and the same other assumptions as mentioned earlier, the trial remains powered at $80 \%$ with a sample size of 500 patients and a total number of 177 events. Sample size calculation was done using EAST Version 6.3.

\section{Interim analysis}

As cardiac safety and the risk of QT prolongation are major concerns during an AE requiring hospital admission, an interim safety and efficacy analysis will be performed as soon as 300 patients have completed their 3-month visit. They will be tested for both efficacy and futility using the alpha- and beta-spending functions of O'Brien-Fleming. ${ }^{41}$ The amount of information will be assessed by the number of observed events over the required number of 177 events to obtain $80 \%$ power. Full details will be available in a statistical analysis plan. These analyses will be performed by an independent safety committee consisting of a cardiologist and a statistician.

\section{Statistical analysis}

All patients randomized will be analyzed according to the intention-to-treat principle. The primary efficacy endpoint 
will be analyzed by means of a logrank test. In addition, a hazard ratio with a $95 \%$ confidence interval from a Cox proportional hazards model will be provided. A serial gatekeeping method ${ }^{42}$ will be used to control the family-wise type I error of the key secondary endpoints that will be addressed in the following hierarchy: number of clinical failures at day 90, symptom CAT score at day 90, and total days of steroid use at day 90. All other secondary outcomes and all analyses performed at day 90 and day 270 will be considered as exploratory. A 5\% significance level will be used. Full details will be available in a statistical analysis plan which will be finalized before the interim analysis.

\section{Data acquisition and analysis}

Data will be collected in an electronic case report form by the local investigators and their dedicated clinical trial assistants, and will be monitored by the central study coordinators of the BACE trial blinded to the randomization code prior to a total database lock. There is no external clinical research organization. Particular attention will go to the adjudication of the primary endpoint as this is a composite one of which all components bear the same weight. For the interim as well as the final analysis, the database will also be checked for outliers after, respectively, the 300th patient has completed his day 90 visit and after the last patient has completed his day 270 follow-up visit. These outliers will be linked to the original on-site documents for revision via queries to the local investigators and clinical trial assistants. After database lock, the randomization code will be broken and the statistical analysis will be performed according to a comprehensive statistical plan which defines primary, secondary, safety, and subgroup analyses.

\section{Conclusion}

This randomized placebo-controlled trial aims to establish an appropriate but more restricted use of azithromycin during and immediately after hospital admission for a severe exacerbation of COPD to overcome the highest risk period for treatment failure, relapse, and death. The proposed intervention will deal not only with short-term in-hospital outcomes but also with the relapse rate during the 3 months after discharge, a period known to have the highest risk for deterioration.

By reducing the dose and the duration of treatment with azithromycin and by targeting the treatment to acute periods with the highest risk for treatment failure, benefits may counterbalance potential side effects of macrolides, which may result in a new treatment strategy for severe AE of COPD. If proven to be safe and effective, a breakthrough in the acute treatment setting will be obtained and the chronic use of high-dose azithromycin in a large and poorly defined population of patients with COPD, with inherent risks and side effects, will be reserved for well-defined subgroups.

\section{Acknowledgments}

The BACE trial is funded by a grant from the Agentschap voor Innovatie door Wetenschap en Technologie (IWT) through the Toegepast Biomedisch onderzoek met een primair Maatschappelijke finaliteit (TBM) program: IWT-TBM number: 130233. KV is supported by the IWT. Financial support for study logistics is also received from Teva Pharma Belgium. Neither IWT nor Teva Pharma Belgium is involved in the study design, in the collection, analysis, and interpretation of data, in the writing of the manuscript, or in the decision to submit the manuscript for publication. The trial is also approved and supported by the Belgian Thoracic Society which provided logistic support for the organization of the investigators' meetings. Special thanks goes to the Clinical Trial Center of UZ Leuven and the contract unit of KU Leuven Research and Development in supporting the financial contracts and legal aspects of the trial organization. Finally, the BACE trial investigators are acknowledged for their participation and the inclusion of the study patients: Vincent Ninane (CHU Saint-Pierre, Brussels, Belgium), Joseph Aumann (Jessa Ziekenhuis, Hasselt, Belgium), Ingel K Demedts (AZ Delta RoeselareMenen, Roeselare, Belgium), Hans Slabbynck (ZNA Middelheim, Antwerpen, Belgium), Eric Marchand (CHU de Mont-Godinne, Yvoir, Belgium), Christel Haenebalcke (AZ Sint-Jan, Brugge-Oostende, Belgium), Rudi Peché (CHU de Charleroi, Charleroi, Belgium), Guy G Brusselle (UZ Gent, Gent, Belgium), Walter Vincken and Shane Hanon (UZ Brussel, Brussels, Belgium), Jean-Louis Corhay (CHU de Liège, Luik, Belgium), Michiel Haerens (AZ Groeninge, Kortrijk, Belgium), Antoine Fremault (Grand Hôpital de Charleroi, Charleroi, Belgium), Tine Lauwerier (Imeldaziekenhuis, Bonheiden, Belgium), Alix Debrock (Sint Augustinus Ziekenhuis, Antwerpen, Belgium), Jan Lamont (Maria Middelares Ziekenhuis, Gent, Belgium), Geert Tits (SintAndriesziekenhuis, Tielt, Belgium), Paul Jordens (OnzeLieve-Vrouwziekenhuis, Aalst, Belgium), Alain Delobbe (Clinique Reine Astrid, Malmedy, Belgium), Jean-Benoît Martinot (Clinique Sainte-Elisabeth, Namur, Belgium).

\section{Author contributions}

Conception and design: WJ and GGB. All authors contributed toward data analysis, drafting and critically revising the paper, gave final approval of the version to be published, and agree to be accountable for all aspects of the work. 


\section{Disclosure}

The authors report no conflicts of interest in this work.

\section{References}

1. Celli BR, Decramer M, Wedzicha JA, et al. An official American Thoracic Society/European Respiratory Society statement: research questions in COPD. Eur Respir Rev. 2015;24:159-172.

2. Dewar M, Curry RW Jr. Chronic obstructive pulmonary disease: diagnostic considerations. Am Fam Physician. 2006;73:669-676.

3. Janssens W, Lehouck A, Decramer M, et al. Vitamin D and the Lung: Mechanisms and Disease Associations. Massachusetts: Springer; 2012: 239-260.

4. Vestbo J, Hurd SS, Agusti AG, et al. Global strategy for the diagnosis, management, and prevention of chronic obstructive pulmonary disease: GOLD executive summary. Am J Respir Crit Care Med. 2013; 187:347-365.

5. Decramer M, De BF, Del PA, et al. Systemic effects of COPD. Respir Med. 2005;99(suppl B):S3-S10.

6. Laveneziana P, Palange P. Physical activity, nutritional status and systemic inflammation in COPD. Eur Respir J. 2012;40:522-529.

7. Brusselle GG, Joos GF, Bracke KR. New insights into the immunology of chronic obstructive pulmonary disease. Lancet. 2011;378: 1015-1026.

8. Wedzicha JA, Seemungal TA. COPD exacerbations: defining their cause and prevention. Lancet. 2007;370:786-796.

9. Wilson R, Sethi S, Anzueto A, et al. Antibiotics for treatment and prevention of exacerbations of chronic obstructive pulmonary disease J Infect. 2013;67:497-515.

10. Seemungal TA, Donaldson GC, Paul EA, et al. Effect of exacerbation on quality of life in patients with chronic obstructive pulmonary disease. Am J Respir Crit Care Med. 1998;157:1418-1422.

11. Wilson R, Anzueto A, Miravitlles M, et al. A novel study design for antibiotic trials in acute exacerbations of COPD: MAESTRAL methodology. Int J Chron Obstruct Pulmon Dis. 2011;6:373-383.

12. Donath E, Chaudhry A, Hernandez-Aya LF, et al. A meta-analysis on the prophylactic use of macrolide antibiotics for the prevention of disease exacerbations in patients with chronic obstructive pulmonary disease. Respir Med. 2013;107:1385-1392.

13. Calverley P, Pauwels R, Vestbo J, et al. Combined salmeterol and fluticasone in the treatment of chronic obstructive pulmonary disease: a randomised controlled trial. Lancet. 2003;361:449-456.

14. Tashkin DP, Celli B, Senn S, et al. A 4-year trial of tiotropium in chronic obstructive pulmonary disease. N Engl J Med. 2008;359:1543-1554.

15. Criner GJ, Bourbeau J, Diekemper RL, et al. Executive summary: prevention of acute exacerbation of COPD: American College of Chest Physicians and Canadian Thoracic Society Guideline. Chest. 2015; 147:883-893.

16. Lopez-Campos JL, Hartl S, Pozo-Rodriguez F, et al. European COPD audit: design, organisation of work and methodology. Eur Respir J. 2013;41:270-276.

17. Sethi S, Murphy TF. Infection in the pathogenesis and course of chronic obstructive pulmonary disease. $N$ Engl J Med. 2008;359:2355-2365.

18. Suissa S, Dell'Aniello S, Ernst P. Long-term natural history of chronic obstructive pulmonary disease: severe exacerbations and mortality. Thorax. 2012;67:957-963.

19. Coeman M, van DY, Bauters F, et al. Neomacrolides in the treatment of patients with severe asthma and/or bronchiectasis: a retrospective observational study. Ther Adv Respir Dis. 2011;5:377-386.

20. Kudoh S, Azuma A, Yamamoto M, et al. Improvement of survival in patients with diffuse panbronchiolitis treated with low-dose erythromycin. Am J Respir Crit Care Med. 1998;157:1829-1832.

21. Saiman L, Marshall BC, Mayer-Hamblett N, et al. Azithromycin in patients with cystic fibrosis chronically infected with Pseudomonas aeruginosa: a randomized controlled trial. JAMA. 2003;290: 1749-1756.
22. Vos R, Vanaudenaerde BM, Verleden SE, et al. A randomised controlled trial of azithromycin to prevent chronic rejection after lung transplantation. Eur Respir J. 2011;37:164-172.

23. Wong C, Jayaram L, Karalus N, et al. Azithromycin for prevention of exacerbations in non-cystic fibrosis bronchiectasis (EMBRACE): a randomised, double-blind, placebo-controlled trial. Lancet. 2012; 380:660-667.

24. Albert RK, Connett J, Bailey WC, et al. Azithromycin for prevention of exacerbations of COPD. N Engl J Med. 2011;365:689-698.

25. Wenzel RP, Fowler AA 3rd, Edmond MB. Antibiotic prevention of acute exacerbations of COPD. N Engl J Med. 2012;367:340-347.

26. Simoens S, Laekeman G, Decramer M. Preventing COPD exacerbations with macrolides: a review and budget impact analysis. Respir Med. 2013;107:637-648.

27. Spagnolo P, Fabbri LM, Bush A. Long-term macrolide treatment for chronic respiratory disease. Eur Respir J. 2013;42:239-251.

28. Giamarellos-Bourboulis EJ, Pechere JC, Routsi C, et al. Effect of clarithromycin in patients with sepsis and ventilator-associated pneumonia. Clin Infect Dis. 2008;46:1157-1164.

29. Johnston SL, Blasi F, Black PN, et al. The effect of telithromycin in acute exacerbations of asthma. N Engl J Med. 2006;354:1589-1600.

30. Global strategy for the diagnosis, management, and prevention of COPD: updated 2015. Global Initiative for Chronic Obstructive Lung Disease; 2015. Available from: http://www.goldcopd.org/guidelinesglobal-strategy-for-diagnosis-management.html. Accessed August 31, 2015.

31. Leuppi JD, Schuetz P, Bingisser R, et al. Short-term vs conventional glucocorticoid therapy in acute exacerbations of chronic obstructive pulmonary disease: the REDUCE randomized clinical trial. JAMA. 2013;309:2223-2231.

32. Walters JA, Wang W, Morley C, et al. Different durations of corticosteroid therapy for exacerbations of chronic obstructive pulmonary disease. Cochrane Database Syst Rev. 2011;10:CD006897.

33. Cheng T, Gong Y, Guo Y, et al. Systemic corticosteroid for COPD exacerbations, whether the higher dose is better? A meta-analysis of randomized controlled trials. Clin Respir J. 2013;7:305-318.

34. Shepard RM, Falkner FC. Pharmacokinetics of azithromycin in rats and dogs. J Antimicrob Chemother. 1990;25(suppl A):49-60.

35. Foulds G, Shepard RM, Johnson RB. The pharmacokinetics of azithromycin in human serum and tissues. J Antimicrob Chemother. 1990; 25(suppl A):73-82.

36. Vos R, Vanaudenaerde BM, Verleden SE, et al. Anti-inflammatory and immunomodulatory properties of azithromycin involved in treatment and prevention of chronic lung allograft rejection. Transplantation. 2012;94:101-109.

37. Niewoehner DE, Erbland ML, Deupree RH, et al. Effect of systemic glucocorticoids on exacerbations of chronic obstructive pulmonary disease. Department of Veterans Affairs Cooperative Study Group. N Engl J Med. 1999;340:1941-1947.

38. Van RH, Raste Y, Louvaris Z, et al. Validity of six activity monitors in chronic obstructive pulmonary disease: a comparison with indirect calorimetry. PLoS One. 2012;7:e39198.

39. Lehouck A, Mathieu C, Carremans C, et al. High doses of vitamin $\mathrm{D}$ to reduce exacerbations in chronic obstructive pulmonary disease: a randomized trial. Ann Intern Med. 2012;156:105-114.

40. Wilson R, Anzueto A, Miravitlles M, et al. Moxifloxacin versus amoxicillin/clavulanic acid in outpatient acute exacerbations of COPD: MAESTRAL results. Eur Respir J. 2012;40:17-27.

41. DeMets DL, Lan KK. Interim analysis: the alpha spending function approach. Stat Med. 1994;13:1341-1352.

42. Bauer P, Rohmel J, Maurer W, et al. Testing strategies in multi-dose experiments including active control. Stat Med. 1998;17:2133-2146. 


\section{Publish your work in this journal}

The International Journal of COPD is an international, peer-reviewed journal of therapeutics and pharmacology focusing on concise rapid reporting of clinical studies and reviews in COPD. Special focus is given to the pathophysiological processes underlying the disease, intervention programs, patient focused education, and self management protocols.

This journal is indexed on PubMed Central, MedLine and CAS. The manuscript management system is completely online and includes a very quick and fair peer-review system, which is all easy to use. Visit http://www.dovepress.com/testimonials.php to read real quotes from published authors.

Submit your manuscript here: http://www.dovepress.com/international-journal-of-chronic-obstructive-pulmonary-disease-journal 